\title{
Methane on a Stepped Surface: Dynamical Insights on the Dissociation of $\mathrm{CHD}_{3}$ on $\mathrm{Pt}(111)$ and $\mathrm{Pt}(211)$
}

Davide Migliorini ${ }^{1}$, Helen Chadwick $^{1}$ and Geert-Jan Kroes $^{1, a}$

${ }^{1}$ Leiden Institute of Chemistry, Gorlaeus Laboratories, Leiden University, P.O. Box 9502, 2300 RA Leiden, The Netherlands ABSTRACT

The simulation of the dissociation of molecules on metal surfaces is a cornerstone for the understanding of heterogeneously catalyzed processes. However, due to high computational demand, the accurate dynamical simulation of the dissociative chemisorption of polyatomic molecules has been limited mostly to flat low-index metal surfaces. The study of surfaces that feature "defected" sites, such as steps, is crucial to improve the understanding of the overall catalytic process due to the high reactivity of undercoordinated sites for this kind of reaction. In this work we have extensively analyzed more than 10000 AIMD trajectories where $\mathrm{CHD}_{3}$ molecule is impinging either on the flat $\mathrm{Pt}(111)$ or on the stepped Pt(211) surface for different initial rovibrational states and collision energies. The results have been compared in order to get insights into the effect of the step in the dissociation of methane. We have found that, despite a large difference in the activation barrier and consequently in reactivity, the geometry of the lowest transition states is very similar on the two surfaces and this results in a similar dissociation dynamics. Furthermore, the trapping observed on the Pt(211) surface can be explained with energy transfer to parallel translational motion induced by the geometry of the slab and by a larger energy transfer to phonons for the stepped Pt(211) surface.

a) Email: g.j.kroes@chem.leidenuniv.nl 


\section{INTRODUCTION}

The dissociative chemisorption of methane on a transition metal surface has been employed, both theoretically ${ }^{1-10}$ and experimentally $y^{11-14}$, as a model system to understand one of the most important steps in steam reforming ${ }^{15}$, a fundamental industrial process which is currently one of the most common ways to produce molecular hydrogen. The $\mathrm{CH}$ bond cleavage on a $\mathrm{Ni}$ or Pt based catalyst is believed to be one of the rate determining steps ${ }^{15}$ of the overall process in the high temperature regime. Due to the large complexity of molecule-surface reactions and to the necessity of treating accurately both the molecule and the metal slab, these systems have always been challenging for appropriate dynamical simulations $^{16-21}$. The large number of degrees of freedom (DOFs) makes density functional theory (DFT) at the generalized gradient approximation (GGA) or meta-GGA level the go-to method for these systems and excludes any higher level of theory, at least for dynamical simulations ${ }^{22}$. Unfortunately, standard GGA and meta-GGA functionals are known for their poor accuracy in the calculation of activation barriers $\left(E_{b}\right)^{22}$ for molecule-surface systems; this has encouraged, in the last few decades, the pursuit of semi-empirical functionals $\mathrm{s}^{3,5,23-25}$ able to compute activation barriers with errors as small as $1 \mathrm{kcal} / \mathrm{mol}$ $(\sim 4.2 \mathrm{~kJ} / \mathrm{mol})$, so-called chemical accuracy.

One of the viable semi-empirical methods to develop an accurate density functional is a variation of the specific reaction parameter (SRP) approach originally proposed by Truhlar and coworkers ${ }^{26}$. In our implementation for molecules on metals this approach consists of mixing two GGA functionals through a mixing parameter $x$ so that the SRP functional is able to reproduce the experimental zero-coverage reaction probability $\left(\mathrm{S}_{0}\right)$ with chemical accuracy. This has been applied successfully to different catalytically relevant systems where a molecule dissociates on a low-index flat metal surface $3,5,23-25$. 
Recently, chemical accuracy has been achieved for $\mathrm{CHD}_{3}$ on $\mathrm{Ni}(111)^{5}$ using the SRP32-vdW functional obtained by mixing the $\mathrm{RPBE}^{27}$ and the $\mathrm{PBE}^{28,29}$ exchange functionals ( $E_{X}^{R P B E}$ and $E_{X}^{P B E}$, respectively) with a mixing parameter $x=0.32$ together with the correlation functional developed by Dion et al. ${ }^{30,31}$ $\left(E_{C}^{v d W-D F}\right)$ that also models van der Waal interactions. The SRP32-vdW functional reads as:

$$
S R P 32-v d W=0.32 \cdot E_{X}^{R P B E}+(1-0.32) \cdot E_{X}^{P B E}+E_{C}^{v d w-D F}
$$

The SRP32-vdW has been successfully transferred from $\mathrm{CHD}_{3}$ dissociation on $\mathrm{Ni}(111)$ to $\mathrm{Pt}(111)$ and, more importantly, it was able to achieve chemical accuracy also on the stepped Pt(211) surface ${ }^{3}$. The transferability of the SRP functional from the flat Pt(111) to the stepped Pt(211) surface suggests that an SRP functional developed for a relatively simple system, such as the flat (111) surface, can be used to obtain accurate reaction barriers on different defected sites of the same metal (see Ref. 3 and references therein). The study of a stepped transition metal surface such as $\mathrm{Pt}(211)$ is highly relevant for heterogeneous catalysis since it is known that under-coordinated sites usually show enhanced reactivity for processes like the dissociative chemisorption of methane ${ }^{32-36}$. Moreover the dissociation of methane on Pt(211) has been previously used to model the overall catalytic process through microkinetic simulations $s^{1,2}$.

In this work we have analyzed 12500 ab-initio molecular dynamics (AIMD) trajectories of $\mathrm{CHD}_{3}$ impinging on both $\mathrm{Pt}(111)$ and $\mathrm{Pt}(211)$ in order to study and compare the dynamical features of the dissociation on the two surfaces focusing on the differences introduced by the presence of the step. The paper is organized as follows: the method used to set up, propagate and analyze the trajectories is reported in Section II, the results are presented and discussed in Section III and the main conclusions are summarized in Section IV. 


\section{METHOD}

Most of the results discussed in this paper have been obtained by analyzing AIMD trajectories of $\mathrm{CHD}_{3}$ impinging on the Pt(111) and on the Pt(211) surfaces. These simulations have been used to compute the zero-coverage reaction probability $\left(\mathrm{S}_{0}\right)$ which has been compared to molecular beam experiments to define and test the SRP32-vdW functional for said systems ${ }^{3}$. The computational details have been extensively discussed in recently published work ${ }^{3}$ and therefore they will only be summarized in this section.

The electronic structure calculations have been performed on a DFT level using the Vienna Ab Initio Simulation Package (VASP) $)^{37-40}$ version 5.3.5. The first Brillouin zone has been sampled with a $4 \times 4 \times 1$ $\Gamma$-centered k-point grid and the basis set includes plane waves with kinetic energy up to $350 \mathrm{eV}$. The core electrons have been represented through projector augmented-wave (PAW) pseudopotentials ${ }^{41,42}$. The Pt(111) surface has been represented using a 5 layers (3x3) supercell slab, consistent with previous work $^{3,6}$. The stepped Pt(211) surface has been modelled using a 4 layer (1x3) supercell. For both surfaces, the slab is separated from its first periodic replica by $13 \AA$ of vacuum. A $0.1 \mathrm{eV}$ Fermi smearing has been used to facilitate the SCF convergence. The set up used for these calculations has been extensively tested and the results of the tests are available in the Supporting Information of Ref. 3.

The AIMD trajectories have been set up in order to reproduce the molecular beam experiments performed by Beck and co-workers ${ }^{3}$ including the surface temperature $\left(T_{s}\right)$ of $500 \mathrm{~K}$ and $650 \mathrm{~K}$ for $\mathrm{Pt}(111)$ and Pt(211) respectively, and modelling the initial kinetic energy and ro-vibrational population of the methane molecules. Note that, even though the simulations have been performed at different temperatures for the two surfaces, previous work suggests that the temperature dependence of the reactivity on Pt is small ${ }^{6,43}$. Quasi-classical trajectories (i.e., classical trajectories where the vibrational 
zero-point energy is imparted to the molecule) have been propagated with a $0.4 \mathrm{fs}$ time-step until dissociation or scattering was observed. A molecule has been considered reacted if a bond was stretched over $3.0 \AA$ and it has been considered scattered if, after the impact, it reached a certain distance above the surface (i.e., $6.0 \AA ̊$ or $6.5 \AA ̊$ for Pt(111) and Pt(211), respectively) while the center of mass (COM) velocity was pointing away from the surface. For the reactive trajectories the time of the dissociation $\left(t_{\text {diss }}\right)$ has been defined as the first time step at which the dissociating bond is as elongated as in the minimum energy transition state $\pm 0.04 \AA$. In this work the molecules that did not reach an outcome within the first 2 ps of propagation have been considered trapped.

The transition state (TS) geometries presented have been computed using the dimer method included in the VASP Transition State Tools package (VTST) ${ }^{44-47}$. Frequency analysis calculations have been performed to confirm that all the TSs reported in this work are true $1^{\text {st }}$ order saddle points (i.e., one and only one imaginary frequency is present) except where stated differently.

The analysis has been carried out on 7000 trajectories for $\mathrm{Pt}(111)$ and on 5500 trajectories for Pt(211) for an initial average incident energy $\left(\left\langle E_{i}\right\rangle\right)$ ranging between 60.7 and $120.1 \mathrm{~kJ} / \mathrm{mol}$ for $\mathrm{Pt}(111)$ and between 58.2 and $107.9 \mathrm{~kJ} / \mathrm{mol}$ for Pt(211). Several dynamics-relevant features have been investigated and the results have been binned and smeared using a sum of Gaussian curves such that, for a sample of $N$ data, the final distribution $\mathcal{F}(\alpha)$ of the observable $\alpha$ is represented as:

$$
\mathcal{F}(\alpha)=\sum_{i}^{N \text { Nins }} \sum_{j}^{N d a t a} \frac{1}{\sqrt{2 \pi \sigma_{G}{ }^{2}}} \cdot \exp \left(-\frac{\left(\text { bin }_{0}+i * \operatorname{binsize}-\operatorname{data}(j)\right)^{2}}{2 \sigma_{G}{ }^{2}}\right)
$$

where the indices $i$ and $j$ run over the bins and the data respectively, $\operatorname{bin}_{0}$ is the first value of $\alpha$ considered for the binning procedure and $\sigma_{G}$ is the standard deviation of the Gaussian used (i.e., the 
broadening). The final distribution is the sum of Gaussians centered on the data values for each element of the dataset. Since we are comparing datasets of different sizes the area of the final distributions is always normalized to 1 . Distributions reported in the same plot always have the same broadening $\sigma_{G}$ and all the distribution parameters are reported in the Supplementary Material (SM).

Using a broadening can improve the quality and the readability of the results but can cause small tails at the edge of the distribution that might assume unphysical values. However this does not affect the interpretation of the results. In order to calculate the distributions all the available trajectories have been used. This means that the distribution takes into account results for different $\mathrm{CHD}_{3}$ vibrational states and average collision energies (see Ref. 3) unless explicitly stated. For some of the observables $\alpha$ the average value is reported together with the standard deviation $\sigma$ and standard error $\sigma_{M}$.

$$
\begin{gathered}
\sigma=\sqrt{\frac{\sum_{i=1}^{N}\left(\alpha_{i}-\langle\alpha\rangle\right)^{2}}{N-1}} \\
\sigma_{M}=\frac{\sigma}{\sqrt{N}}
\end{gathered}
$$

Here, $N$ is the sample size and $\langle\alpha\rangle$ is the sample average.

\section{RESULTS AND DISCUSSION}

Platinum is an fcc metal and its (111) surface shows the well-known hexagonal lattice structure. The $\mathrm{Pt}$ (211) surface is composed of a 3 atoms wide (111)-like terrace and a (100)-like step. In order to analyze the dynamics on the $\mathrm{Pt}(211)$ three non-equivalent sites have been defined: edge (on the step edge), middle (the row of atoms in the middle of the terrace) and bottom (the row of atoms on the low 
end of the step). Moreover some of the analysis is referenced to facets instead of sites considering the step (i.e., the (100) facet) and the terrace (i.e., the (111) facet). The side and top views of the surfaces are shown in Fig. 1 where the atoms are reported in their equilibrium positions and the step facet is highlighted in green for the $\mathrm{Pt}(211)$ surface. The impact (reaction) site is defined as the nearest site (i.e., bottom, middle or edge) in the $x y$ plane at the closest approach (reaction) time. Molecules that hit the surface or dissociate at an $x$ coordinate between the edge and the bottom site (green shaded area in Fig. 1) are considered step facet events. It has been shown in previous work ${ }^{3}$ that the reactivity on the stepped surface happens almost completely through the dissociation on top of the step edge atom. Therefore the analysis of reacted trajectories has been carried out by looking at the different behavior of molecules impacting on the different surface sites (i.e., edge, middle and bottom atoms). For nonreactive trajectories the differences in the dynamics upon impacting on the (111)-like terrace facet or on the (100)-like step facet have also been considered.

On the Pt(211) surface trapping has been observed and the average trapping time has been estimated to be about $40 \mathrm{ps}^{3}$ (i.e., $43 \mathrm{ps}$ ), which is too short to be detected experimentally and too long to be simulated with an expensive method like AIMD. For the previously published work the trapped trajectories have been propagated for 1 ps (or 2 ps in a few test cases). Therefore, to ensure a fair analysis, in this work all the trapped trajectories have been propagated up to 2 ps. Out of the 69 trajectories that were trapped after 1 ps 35 have scattered upon further propagation. However this does not necessarily affect the main conclusions of earlier work since a large number of trapped trajectories are still present even after 2 ps of propagation, especially at low incidence energy. This also suggests that, in order to be trapped (i.e., characterized by some average trapping time), the molecules need some time to equilibrate with the surface.

To understand the dynamics of the $\mathrm{CHD}_{3}$ on the different surfaces, the number of bounces has been counted for all the trajectories, where a bounce is defined as two sign-changes of the COM velocity 
along the direction parallel to the macroscopic surface normal $(z)$. As observed for other metal surfaces, the $\mathrm{CHD}_{3}$ dissociative chemisorption is usually a "sudden" process: on both platinum surfaces the molecules react on the first impact and without bouncing. This is also true for almost all the scattered trajectories on $\mathrm{Pt}$ (111) (only 5 out of more than 6500 perform one and only one bounce) and for most of the scattered trajectories on Pt(211). On Pt(211) (see Fig. 2) we observe few trajectories bouncing either one or two times before scattering and that the trapped trajectories bounce between one and four times during the propagation.

\section{A. Transition States}

Several transition states have been located on the stepped $\mathrm{Pt}(211)$ surface by performing calculations using the dimer method ${ }^{44-47}$. All the TSs presented are real 1st order saddle points as confirmed by a normal mode analysis unless stated differently. The energy (i.e. the barrier height, $E_{b}$ ), the length of the dissociating $\mathrm{CH}$ bond $(\mathrm{r})$ and the $\theta, \beta$ and $\gamma$ angles have been determined. $\theta$ is the angle between the dissociating bond and the surface normal (i.e., $z)$ and $\beta$ is the angle between the umbrella axis $(\vec{a})$ and the surface normal, where $\vec{a}$ is defined as the vector going from the geometric center of the umbrella hydrogens to the carbon atom (Fig. 3). $\gamma$ is the angle between the dissociating $\mathrm{CH}$ bond and the $\vec{a}$ axis. The $\gamma$ angle describes the internal geometry of the TS while $\beta$ and $\theta$ describe the geometry of the molecule with respect to the macroscopic surface normal. All the results, compared to the minimum energy TS for the flat Pt(111) surface, are reported in Table I including all the TS geometries (also represented in Fig. 4) and the studied angles (also shown in Fig. 3). The angle $\alpha$ (sketched in Fig. 3 and reported in Table I) describes the orientation of the dissociating bond in the $x y$ plane for the Pt(211) surface and it will be discussed later in this paper. 
Table I. Comparison of the TSs found on the two platinum surfaces. For each TS is reported: the surface on which it has been found, the Label as in Fig. 4 , the name of the TS, the $\theta, \beta, \gamma$ and $\alpha$ angles, the length of the dissociating bond $(\mathrm{r})$ in $\AA$ and the energy barrier $\left(E_{b}\right)$ in $\mathrm{kJ} / \mathrm{mol}$. Note that if the energy barrier is followed by a star $\left({ }^{*}\right)$ the TS reported still has a second imaginary frequency (i.e., it is not a real $1^{\text {st }}$ order saddle-point).

\begin{tabular}{|c|c|c|c|c|c|c|c|c|}
\hline Surface & Label & Name & $\theta\left[^{\circ}\right]$ & $\beta\left[^{\circ}\right]$ & $\gamma\left[^{\circ}\right]$ & $\alpha\left[^{\circ}\right]$ & $\mathrm{r}[\AA ⿻$ & $E_{b}[\mathrm{~kJ} / \mathrm{mol}]$ \\
\hline $\mathrm{Pt}(111)$ & $\mathrm{A}$ & - & 133.4 & 168.3 & 34.8 & - & 1.55 & 78.6 \\
\hline $\mathrm{Pt}(211)$ & $\mathrm{B}$ & Edge2Edge-B1 & 132.9 & 166.0 & 33.1 & 85.0 & 1.53 & 53.9 \\
\hline $\mathrm{Pt}(211)$ & $\mathrm{C}$ & Edge2Mid-H2 & 115.6 & 145.6 & 30.0 & 181.3 & 1.60 & 57.1 \\
\hline $\mathrm{Pt}(211)$ & $\mathrm{D}$ & Edge2Mid-H1 & 113.7 & 145.9 & 32.2 & 179.4 & 1.62 & $57.7^{*}$ \\
\hline $\mathrm{Pt}(211)$ & $\mathrm{E}$ & Edge2Bot-B1 & 122.9 & 156.8 & 33.9 & 0.5 & 1.65 & $65.1^{*}$ \\
\hline $\mathrm{Pt}(211)$ & $\mathrm{F}$ & Mid2Bot-B1 & 145.8 & 171.8 & 35.0 & 226.3 & 1.55 & 97.9 \\
\hline $\mathrm{Pt}(211)$ & $\mathrm{G}$ & Mid2Mid-B1 & 130.2 & 158.5 & 34.9 & 72.1 & 1.57 & 98.0 \\
\hline $\mathrm{Pt}(211)$ & $\mathrm{H}$ & Mid2Edge-B1 & 119.0 & 151.7 & 33.4 & 25.3 & 1.56 & 96.4 \\
\hline
\end{tabular}

For Pt(211), four TSs have been found on top of a step edge atom, with a different orientation of the dissociating $\mathrm{CH}$ bond. These have been labeled according to the positions of the carbon and of the dissociating hydrogen: Edge2Edge-Bridge (Edge2Edge-B) dissociating parallel to the step edge (Fig. 4B), Edge2Middle-Hollow (Edge2Mid-H) dissociating on a hollow site towards the middle row of the terrace (Figs. 4C and 4D) and Edge2Bottom-Bridge (Edge2Bot-B) dissociating towards a bridge site on the (100) step site (Fig. 4E). Note that the two TS depicted in Fig. 4C and 4D are related by a hindered rotation about the umbrella axis. Three TSs have been found on the middle terrace atom dissociating towards different bridge sites and they have been labeled as Middle2Bottom-Bridge (Mid2Bot-B), Middle2Middle-Bridge (Mid2Mid-B) and Middle2Edge-Bridge (Mid2Edge-B) according to which site they are dissociating towards (Figs. 4F, 4G and 4H, respectively). The numbers, either 1 or 2 , at the end of a TS name identify the eclipsed or the staggered orientation of the umbrella relative to the dissociating $\mathrm{CH}$ bond, respectively. Note that the TSs depicted in Figs. 4D and 4E still have a second small imaginary frequency (i.e., they are not real $1^{\text {st }}$ order saddle points. See Table I).

There is a clear difference in the $E_{b}$ between the step edge atom and on the terrace middle atom (i.e., $\approx$ $39 \mathrm{~kJ} / \mathrm{mol}$ on average) regardless of the molecular orientation: the TSs centered on the step edge atom 
have very similar energies and the same is true for the TSs centered on the terrace middle atom. The step edge site is more reactive than the flat $\mathrm{Pt}(111)$ surface while terrace middle sites show barriers even larger than on the ideal flat surface $\left(78.6 \mathrm{~kJ} / \mathrm{mol}\right.$, Table I). Despite this large range of $E_{b}$ the TSs are geometrically similar: all of them have a dissociating bond length between 1.53 and $1.65 \AA$ and an angle $\gamma$ between the dissociating bond and $\vec{a}$ between 30 and $35^{\circ}$. As expected $\beta$ and $\theta$ show more variability because they depend on the molecular geometry as well as on the surface orientation with respect to the reference frame ( $z$ axis). $\beta$ and $\theta$ can be compared for the TS on $\mathrm{Pt}(111)$ and the lowest energy TS on Pt(211) (i.e., Edge2Edge-B1) and, for both the angles, they have the same values within less than $2.5^{\circ}$.

\section{B. Energy Transfer to Parallel Motion}

In Fig. 5 the COM lateral displacement $\left(\delta_{C O M}\right)$ has been reported for all the trajectories, where $\delta_{C O M}$ has been computed as the difference between the COM $x y$ position at the initial and at the reaction step (for dissociative events) or at the step of $1^{\text {st }}$ closest approach (for scattered and trapped trajectories). For the flat surface the reactive events happen in a "sudden" fashion where the molecule does not change its position in the $x y$ plane significantly, as they show $\left\langle\delta_{C O M}\right\rangle$ smaller than $0.09 \AA$ (Fig. $5 \mathrm{~A}$ ). The same is true for the molecules reacting on the step edge of Pt(211) (Fig. 5B). Scattered and trapped molecules on $\mathrm{Pt}(211)$ show a significantly larger $\left\langle\delta_{C O M}\right\rangle$ that can go up to $0.25 \AA$ for molecules trapped after impacting on the step facet (all the data are reported in the SM). This is due to the fact that, even though all molecules start with a COM velocity aligned with the $z$ axis, the topology of the surface can induce energy transfer from the translational motion in the $z$ direction to the motion along the surface (energy transfer to parallel motion). In order to study this phenomenon, the distance travelled in the $x y$ plane has been computed (for each individual trajectory) as: 


$$
\begin{gathered}
d_{x y}=\sum_{t=1}^{T} \sqrt{\left(x_{t}-x_{t-1}\right)^{2}+\left(y_{t}-y_{t-1}\right)^{2}} \\
d_{\alpha=x, y}=\sum_{t=1}^{T}\left|\alpha_{t}-\alpha_{t-1}\right|
\end{gathered}
$$

Here, $d_{x y}$ is the distance travelled in the $x y$ plane and $d_{\alpha}$ is the distance travelled in the $\alpha$ direction (i.e., $\alpha=x, y)$, and $t$ is the time step. T is the total number of time steps. Note that $d_{x y}$ and $d_{\alpha}$ are dependent on the time step ( $0.4 \mathrm{fs}$ ) and on the total propagation time (i.e., the number of time steps $T$ ). On Pt(211), $x$ and $y$ are perpendicular and parallel to the step edge, respectively. If we consider $d_{x y}$, reported in Fig. 6, it is noticeable how the molecules scattered from the step can travel significantly more than the ones scattered from the flat surface (Fig. 6A and 6B). On average we observe $\left\langle d_{x y}\right\rangle=0.8$ $\AA ̊$ on $\mathrm{Pt}(111)$ and $\left\langle d_{x y}\right\rangle=2.8 \AA$ on $\mathrm{Pt}(211)$ but on the stepped surface scattered molecules have been observed to travel up to $30 \AA$.

If we look at the trajectories trapped on the Pt(211) surface (Fig. 6C) we can see that the molecules can travel considerable distances in our simulations (i.e., up to almost $50 \AA ̊$ ) especially in the direction perpendicular to the step edge (i.e., along $x$ ). Note that for these trajectories the propagation has been stopped after 2 ps and the more we would propagate them the further they would travel. This is due to the large velocity the trapped molecules have in the $x y$ plane.

The distribution of the average velocity in $x y$ upon trapping $\left(\left\langle V_{x y}\right\rangle^{\text {trap }}\right)$ is reported in Fig. 6D. $\left\langle V_{x y}\right\rangle^{\text {trap }}$ has been computed as: 


$$
\left\langle v_{x y}\right\rangle^{\text {trap }}=\sum_{t=1}^{N^{\text {trap }}} \frac{\sqrt{v_{x(t)}^{2}+v_{y(t)}^{2}}}{N^{\text {trap }}}
$$

$\left\langle V_{x y}\right\rangle^{t r a p}$ is averaged over the total of $N^{\text {trap }}$ time steps. Here $t$ ranges from the first impact with the surface to the last step of the propagation (i.e., when the molecule is considered trapped). The velocity distribution is centered on $\left\langle V_{x y}\right\rangle^{\text {trap }}=0.014 \AA / \mathrm{fs}$ which means that during the estimated trapping time of 43 ps, those molecules can travel, on average, as far as $593 \AA$ and this goes up to $642 \AA$ if we consider only the molecules trapped after impinging on the step. This suggests that the trapped molecules have enough time to explore a large portion of the surface, increasing their chance of finding a favorable orientation to react or even a higher order defect with a lower dissociation barrier, such as kinks.

The importance of the energy transfer from motion normal to the surface to motion parallel to the surface can be quantified by calculating the amount of kinetic energy that molecules have in the $x y$ plane after the impact with the surface $\left(K_{x y}\right)$. To ensure a fair comparison, $K_{x y}$ has been calculated at the end of the trajectory for scattered molecules and at the first outer turning point for trapped molecules. In Fig. $7 K_{x y}$ is reported for scattered and trapped molecules on Pt(211) distinguishing between molecules which impact on the step facet and on the terrace facet. As expected both trapped and scattered molecules show large values of $K_{x y}$. Moreover, the step site is more efficient than the terrace in promoting energy transfer to the parallel motion, probably because it makes a larger angle with the normal to the macroscopic surface. In Fig. $8\left\langle K_{x y}\right\rangle$ is reported as a function of the initial average collision energy of the molecules $\left(\left\langle E_{i}\right\rangle\right)$. The energy transfer to parallel translational motion increases with $\left\langle E_{i}\right\rangle$ and confirms that the step induces more energy transfer to the parallel motion than the terrace. The values of $\left\langle K_{x y}\right\rangle$ for the trapped trajectories show large error bars due to the small sample 
size but the results suggest that the trapped molecules show a larger energy transfer to the parallel motion than the scattered ones at low $\left\langle E_{i}\right\rangle$ (Fig. 8). In turn, this suggests that, especially at low $\left\langle E_{i}\right\rangle$, energy transfer to parallel motion at the step contributes to the trapping.

Out of a total of 34 trapped trajectories 25 of them impacted on the step while only 9 on the terrace. If we weight these results according to the step:terrace area ratio (i.e., 1:1.8) we find that the trapping probability is 5 times larger if the molecule impacts on the step than on the terrace. The $x z$ projection of the first part of the trapped trajectories is reported in Fig. 9. In Fig. 10 is reported the distribution of the average velocity in $x$ for the trapped molecules $\left(\left\langle v_{x}\right\rangle^{t r a p}\right)$. Again, these results suggest that one of the main reasons for the trapping we observe on $\mathrm{Pt}(211)$ is due to the geometry of the surface: the molecules that hit the step undergo energy transfer to parallel translational motion and start travelling in the positive $x$ direction. Most of the molecules impacting on the terrace travel in the negative $x$ direction.

\section{Energy Transfer to Surface Phonons}

The energy transfer from the molecules to the surface phonons $\left(E_{T}\right)$ is reported in Fig. 11. All the scattered molecules transfer roughly the same amount of energy to the phonons independently from the Pt surface on which they impact; on average molecules that hit the $\mathrm{Pt}(111)$ and the $\mathrm{Pt}(211)$ surface show $\left\langle E_{T}\right\rangle=15.5 \pm 0.2$ and $13.9 \pm 0.2 \mathrm{~kJ} / \mathrm{mol}$ respectively. Note that $\mathrm{Pt}(111)$ shows a somewhat larger $\left\langle E_{T}\right\rangle$ but the AIMD trajectories have been performed over a larger range of $\left\langle E_{i}\right\rangle$. This can be seen in Fig. $11 \mathrm{~B}$ where $E_{T}$ is reported as a function of the incident energy: the linear regression for the two sets of data show the same slope (i.e., 0.165) and very similar intercepts (i.e., $0.914 \mathrm{~kJ} / \mathrm{mol}$ and $1.400 \mathrm{~kJ} / \mathrm{mol}$ for $\mathrm{Pt}(111)$ and $\mathrm{Pt}(211)$, respectively). Note that the difference of $0.486 \mathrm{~kJ} / \mathrm{mol}$ in the intercept is smaller than the average error for both surfaces (i.e., $0.527 \mathrm{~kJ} / \mathrm{mol}$ and $0.514 \mathrm{~kJ} / \mathrm{mol}$ for $\mathrm{Pt}(111)$ and $\mathrm{Pt}(211$ ), 
respectively). The small number of trapped trajectories reduces the statistical significance of their analysis. However the results suggest that, especially at low $\left\langle E_{i}\right\rangle$, some trajectories can undergo a larger energy transfer to the surface phonons (i.e., $\left\langle E_{T}\right\rangle=23.2 \pm 1.9 \mathrm{~kJ} / \mathrm{mol}$ ) which would increase the probability of being trapped. This suggests that energy transfer to the phonons contributes to the trapping on Pt(211).

The $E_{T}$ has been calculated approximately from the molecular kinetic $(K)$ and potential $(V)$ energy. The initial configuration $(i)$ is always the first step of the dynamics. For the scattered molecule, the final configuration $(f)$ is taken from the last step of the dynamics while for the trapped molecule we considered the $E_{T}$ upon first impact with the surface and therefore the final configuration is taken from the first outer turning point configuration. This means that, in the initial and in the final configurations, the molecules might not be at the same distance from the slab. Therefore the energy transfer has been estimated using Eq. 8a, which includes a correction for the interaction energy $(I)$. $I$ has been computed using Eq. $8 \mathrm{~b}$ and subtracting the absolute energy of the isolated $\mathrm{CHD}_{3}$ and of the bare slab from the energy of the system containing both the molecule and the metal surface.

$$
\begin{gathered}
E_{T}=-\left[\left(K_{f}+V_{f}+I_{f}\right)-\left(K_{i}+V_{i}+I_{i}\right)\right] \\
I=V_{\text {slab }+ \text { CHD } 3}-V_{\text {slab }}-V_{C H D 3}
\end{gathered}
$$

\section{Reaction Site and Dissociation Geometry}

On Pt(211) the vast majority of the reactive events happen on the step edge. The reaction site has been studied by measuring the distance in the $x y$ plane from the center of mass of the molecule to the closest atom in the first layer and reporting the distributions for all the trajectories simulated. Results 
are reported in Fig. 12A for $\mathrm{Pt}(111)$ and in Fig. 12B for $\mathrm{Pt}(211)$ for the reacted molecule at $\mathrm{t}=0$ and at the time of the dissociation ( $\left.t=t_{\text {diss }}\right)$. For both surfaces the reaction happens preferentially close to top sites (i.e., atoms in the first layer) but for the stepped surface a considerable number of molecules dissociates further than $1 \AA$ away from the closest Pt atom.

The effect of the orientation of the molecules on reaction has been analyzed by monitoring the $\beta$ (Fig. 13), the $\theta$ (Fig. 14) and the $\gamma$ (Fig. 15) angles throughout the dynamics and investigating their dependence on $\left\langle E_{i}\right\rangle$ (Fig. 16). These three angles describe the orientation of the umbrella axis $(\beta)$ and of the dissociating bond $(\theta)$ with respect to the surface normal and the angle between the dissociating bond and the umbrella axis $(\gamma)$ (see Fig. 3). The angles have been computed at the start of the trajectories $(\mathrm{t}=0)$ and at the time of the dissociation $\left(\mathrm{t}=\mathrm{t}_{\text {diss }}\right)$ for the reactive trajectories. For both $\theta$ and $\beta$ the initial distribution considering all the molecules simulated is a sine distribution proving the accurate random sampling of the initial conditions.

Comparing the $\mathrm{Pt}(111)$ to the $\mathrm{Pt}(211)$ results one can see that the dissociation dynamics on the two surfaces are qualitatively very similar: the two known key aspects of the dissociation of $\mathrm{CHD}_{3}$ on $\mathrm{Pt}$ and $\mathrm{Ni}$ are the pre-orientation of the dissociating bond $\mathrm{d}^{4,5}$ and the umbrella reorientation ${ }^{4}$. The distributions of $\theta$ reported in Fig. 14 show that only bonds initially pointing towards the surface (i.e. close to the TS value of $\theta \approx 133^{\circ}$ ) can dissociate and that the angular acceptance range for the dissociation on the $\mathrm{Pt}(211)$ surface is broader as the final $\theta$ distributions are less localized and exhibit larger $\sigma$ values (see Fig. 14 and Table II). The results for $\beta$ (see Fig. 13) confirm the important role of the umbrella reorientation ${ }^{4}$ for $\mathrm{CHD}_{3}$ on $\mathrm{Pt}(111)$, and extend this confirmation to the reaction on the stepped $\mathrm{Pt}(211)$ surface as well. In order to react, the molecule not only needs to approach the surface with the $\mathrm{CH}$ bond pre-oriented towards the surface, but in the meantime the umbrella has to swing upwards increasing the value of $\beta$. In previous work ${ }^{4}$ Füchsel et al. studied the dissociation of $\mathrm{CHD}_{3}$ on $\operatorname{Pt}(111)$ and, for $\left\langle E_{i}\right\rangle$ between 49 and $84 \mathrm{~kJ} / \mathrm{mol}$, they observed that the center of the distributions of $\theta$ do not shift during 
the reaction. Extending the range of initial average kinetic energy we do observe a dependence of $\theta$ and of $\gamma$ on $\left\langle E_{i}\right\rangle$ at the time of the reaction (see Fig. 16) that can be explained by considering that, since the hydrogen (or deuterium) atom is oriented towards the surface, the dissociating bond bends away from the surface upon impacting on the slab proportionally to the COM velocity and thus results in a decrease of $\theta$ and in an increase of $\gamma$ (since $\beta$ does not depend on $\left\langle E_{i}\right\rangle$ ).

Table II. Average value, standard deviation $(\sigma)$ and standard error $\left(\sigma_{M}\right)$ for $\beta, \theta$ and $\gamma$ at the time of the dissociation as reported in Figs. 13, 14 and 15, respectively.

\begin{tabular}{|l|c|c|c|c|c|c|}
\hline Surface & \multicolumn{2}{|c|}{$\beta\left[^{\circ}\right]$} & \multicolumn{2}{c|}{$\theta\left[{ }^{\circ}\right]$} & \multicolumn{2}{c|}{$\gamma\left[{ }^{\circ}\right]$} \\
\hline & $\langle\beta\rangle \pm \sigma_{M}$ & $\sigma$ & $\langle\theta\rangle \pm \sigma_{M}$ & $\sigma$ & $\langle\gamma\rangle \pm \sigma_{M}$ & $\sigma$ \\
\hline $\operatorname{Pt}(111)$ & $149.9 \pm 0.6$ & 12.1 & $123.6 \pm 0.5$ & 10.2 & $33.8 \pm 0.6$ & 13.0 \\
\hline $\operatorname{Pt}(211)$ & $141.2 \pm 1.1$ & 16.7 & $117.3 \pm 1.0$ & 15.0 & $31.9 \pm 0.8$ & 12.2 \\
\hline
\end{tabular}

Fig. 16 also shows that, at the moment of the dissociation, $\beta$ and $\theta$ (Figs. 16A and 16B) are smaller for $\mathrm{Pt}$ (211) than for Pt(111) while $\gamma$ is very similar (Fig. 16C). This suggests that at the moment of the reaction the internal molecular geometry is the same on the two surfaces. However the molecules react, on average, more parallel to the macroscopic surface for the stepped $\mathrm{Pt}(211)$. This can be explained by looking at the $\theta$ angle of the TSs on the two surfaces. All the TSs on Pt(111) show $\theta \approx 133^{\circ}$ (see Table III in Ref. 48) which is the same value of the lowest $E_{b}$ TS on Pt(211) (Edge2Edge-B1 in Fig. 4B and Table I). However, the TSs perpendicular to the step edge on $\mathrm{Pt}(211)$ (i.e., C, D and E in Table I) show values of $\theta$ about $10-19^{\circ}$ smaller and this effectively broadens the angular acceptance range for the dissociation on the stepped surface. The TSs C, D and E dissociate in the direction perpendicular to the step edge and, even though they show larger $E_{b}$, they play a big role in the dissociative process. In Fig. 17 the distribution of the angle $\alpha$ is plotted at $\mathrm{t}=0$ and the moment of the dissociation for molecule reacting on the step edge atom. $\alpha$ is defined as the counter-clockwise angle between $x$ and the projection of the dissociating bond on the $x y$ plane. Therefore, $\alpha=0^{\circ}$ corresponds to a dissociation in the $x$ direction and $\alpha=90^{\circ}$ corresponds to the $y$ direction (i.e., perpendicular and parallel to the step edge, respectively). 
Even though the minimum energy barrier TS occurs at $\alpha \approx 0^{\circ}$ (and, equivalently, at $\alpha \approx 270^{\circ}$ ), most of the reactions on the step edge atom happen for $\alpha \approx 180^{\circ}$. This can be partially explained taking into account that the orientation of the molecules is sampled as $\sin (\theta)$ which makes $\theta \approx 114^{\circ}$ (i.e., the ideal value for $\alpha=180^{\circ}$ ) oversampled with respect to $\theta \approx 133^{\circ}$ (i.e., the ideal value for $\alpha=90^{\circ}$ ) (see Table I).

\section{SUMMARY AND CONCLUSION}

In this work we have analyzed a total of 12500 trajectories where $\mathrm{CHD}_{3}$ is impinging either on the flat $\mathrm{Pt}$ (111) or on the stepped Pt(211) surface. Moreover several different TSs have been found on the $\mathrm{Pt}(211)$ surface. Despite all the TSs found having a very similar molecular geometry, the ones located on a step edge atom of the Pt(211) surface show a significantly lower energy barrier while the ones on the terrace middle atom have larger barrier heights, even larger than for the flat Pt(111) surface. On both surfaces the dissociation has been shown to proceed through a very similar mechanism where the dissociative $\mathrm{CH}$ bond needs to be pre-oriented towards the surface and the methyl umbrella rotates upwards to promote the reaction. However the stepped $\mathrm{Pt}(211)$ has a broader angular acceptance range for the dissociative chemisorption and the molecules react on average more parallel to the surface. The stepped Pt(211) surface can trap molecules due to a larger energy transfer to phonons and to geometrypromoted energy transfer to parallel translational motion. The large velocity in the direction parallel to the surface of the trapped trajectories suggests that they can travel long distances, on average more than $600 \AA$ for step impacts with trapping times > 1 ps, while bouncing on the surface. This implies that, in experiments, the trapped molecule has time to explore the surface and therefore it has an increased probability of reacting by finding the most favorable geometry or even a higher order defect, such as kinks. 


\section{SUPPLEMENTARY MATERIAL}

The Supplementary Material contains Tables with the averages, the standard deviations and the binning parameters for all the data reported in the paper.

\section{ACKNOWLEDGMENTS}

This work has been financially supported by the European Research Council through an ERC2013 advanced grant (Nr. 338580), by the Nederlandse Organisatie voor Wetenschappelijk Onderzoek (NWO-

CW), by a grant of computing time from NWO-EW and by the Swiss National Science Foundation (grant Nr. P300P2-171247).

The authors thank Nick Gerrits for the useful discussions. 


\section{FIGURES}
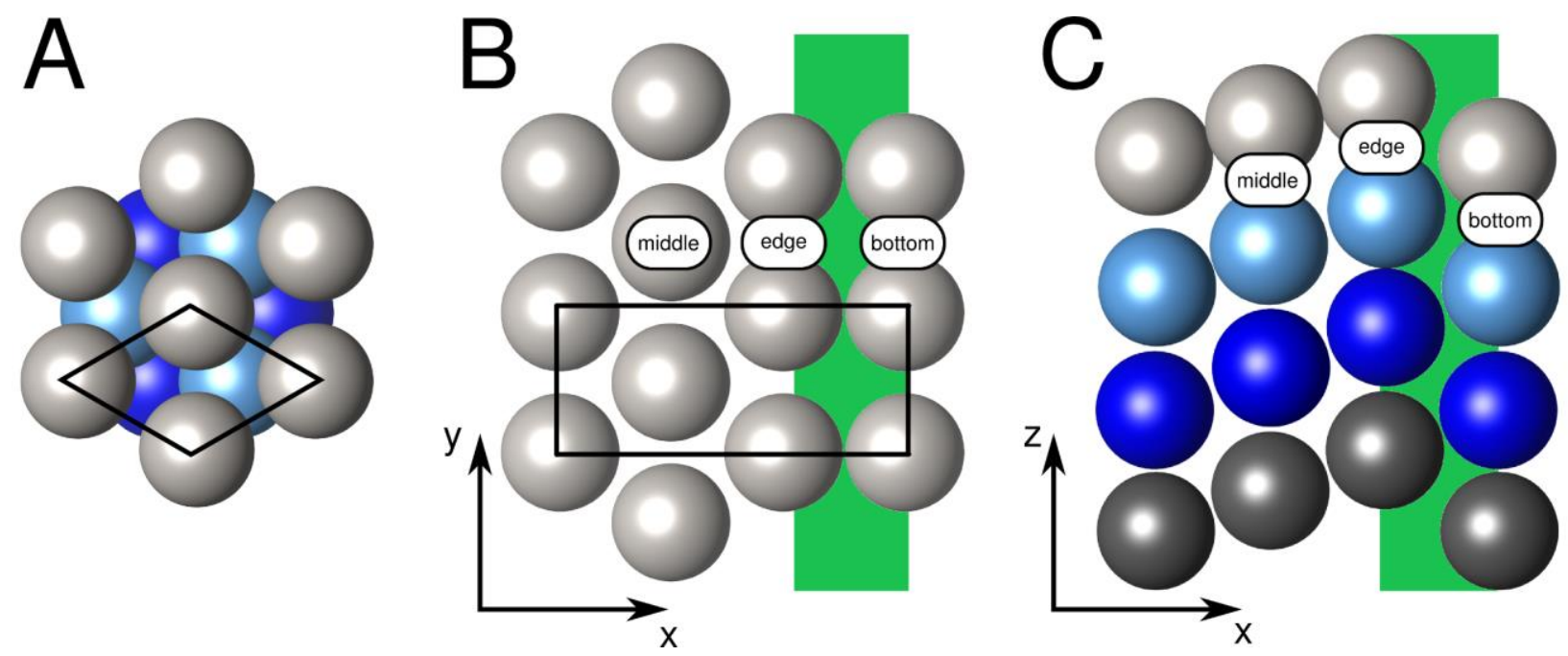

Figure 1. Panel A: top view of the Pt(111) surface. Panel B: top view of the first layer of the Pt(211)

surface. Panel C: side view of the Pt(211) surface. For both the surfaces the atoms are reported in their equilibrium position. The unit cell is marked in black and different layers are reported in different colors. For the Pt(211) surface the three rows of atoms in the unit cell are called edge, middle and bottom according to their position: edge is on top of the step edge, middle is in the middle of the terrace, and bottom is on the low side of the step. The (100) step is shaded in green while the remainder of the surface is the (111) terrace. 

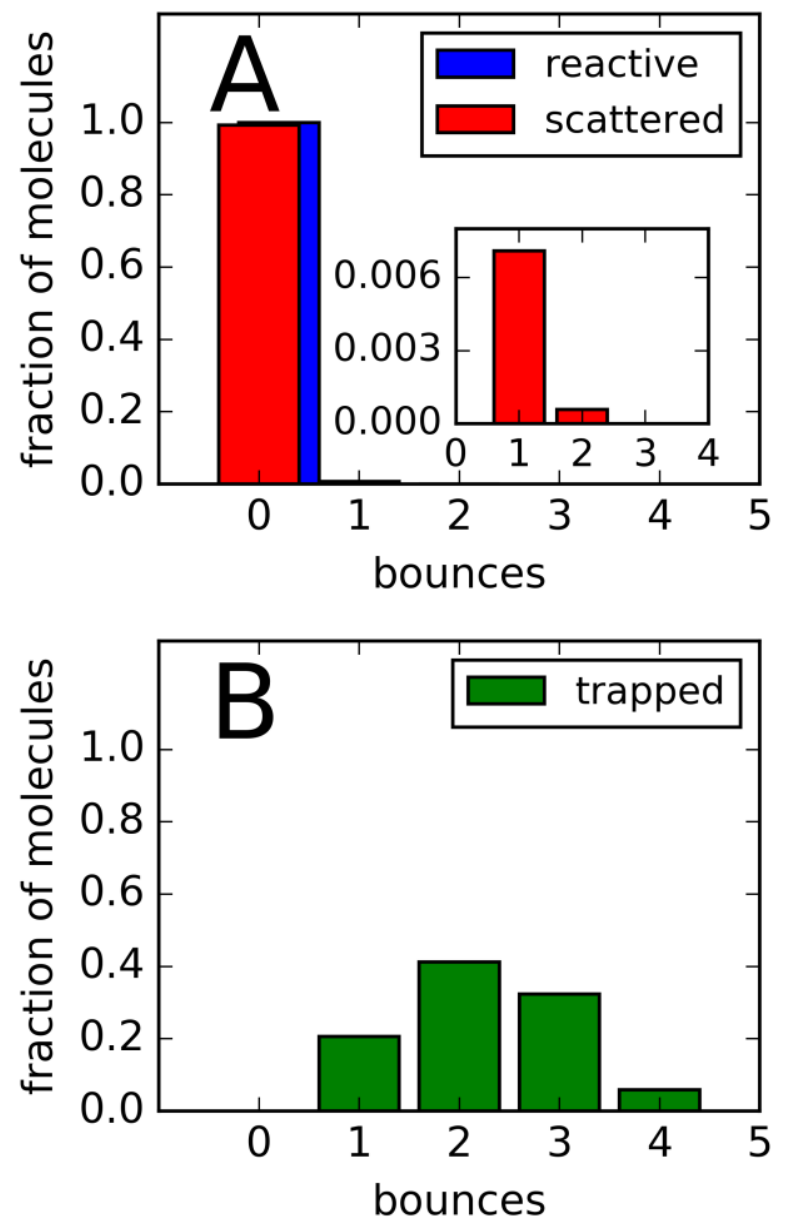

Figure 2. Number of bounces for the molecules impinging on the Pt(211) surface. Panel A reports in red and blue the fraction of scattered and reacted trajectories, respectively. The inset in Panel $A$ is a magnification for the few trajectories that bounce before scattering. Panel B reports the results for the trapped trajectories on $\mathrm{Pt}(211)$. 


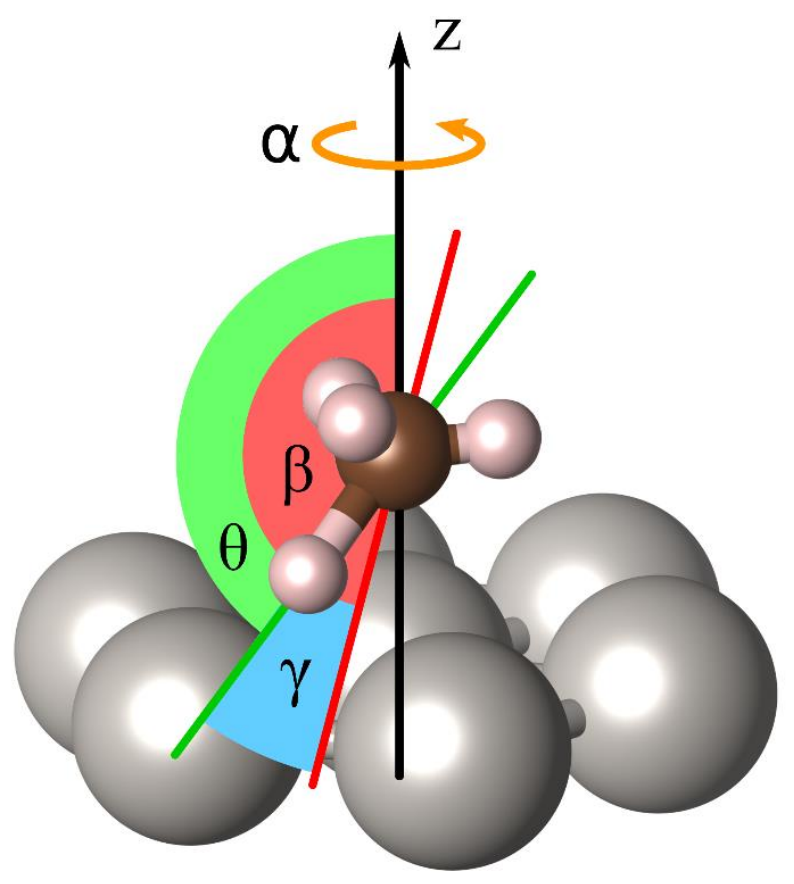

Figure 3. Sketch of the angles studied: $\theta$ (green shade) is the angle between the $\mathrm{CH}$ bond (green line) and the $z$ axis, $\beta$ (red) is the angle between the the umbrella axis $\vec{a}$ (red line) and the $z$ axis (i.e., the macroscopic surface normal) and $\gamma$ (blue) is the angle between the $\mathrm{CH}$ bond and the umbrella axis $\vec{a}$. The angle $\alpha$ (orange) represents the orientation of the dissociating bond in the $x y$ plane. 

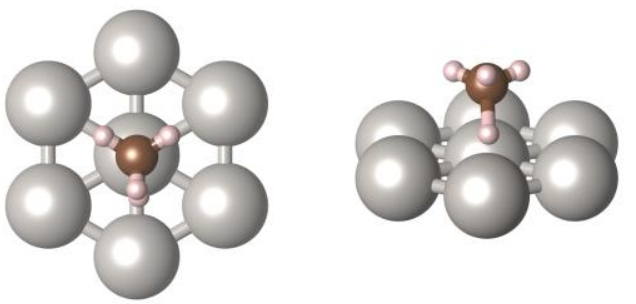

A
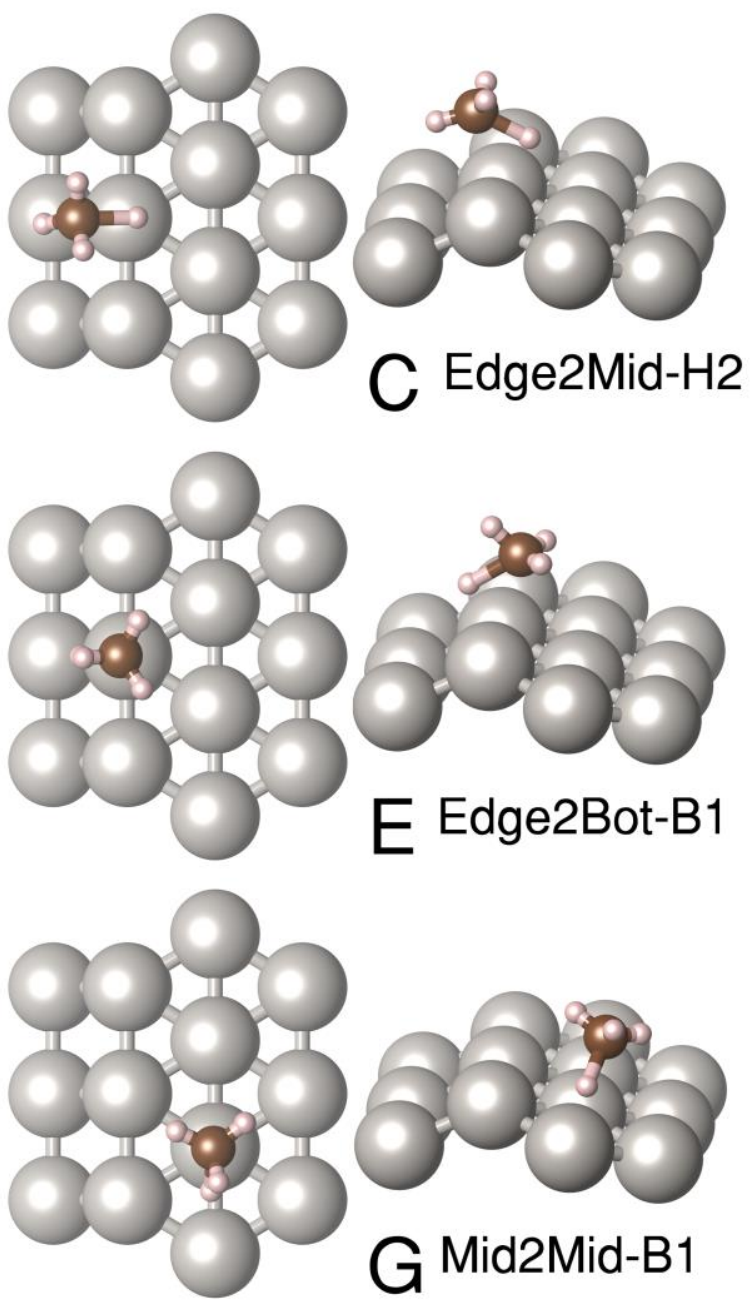
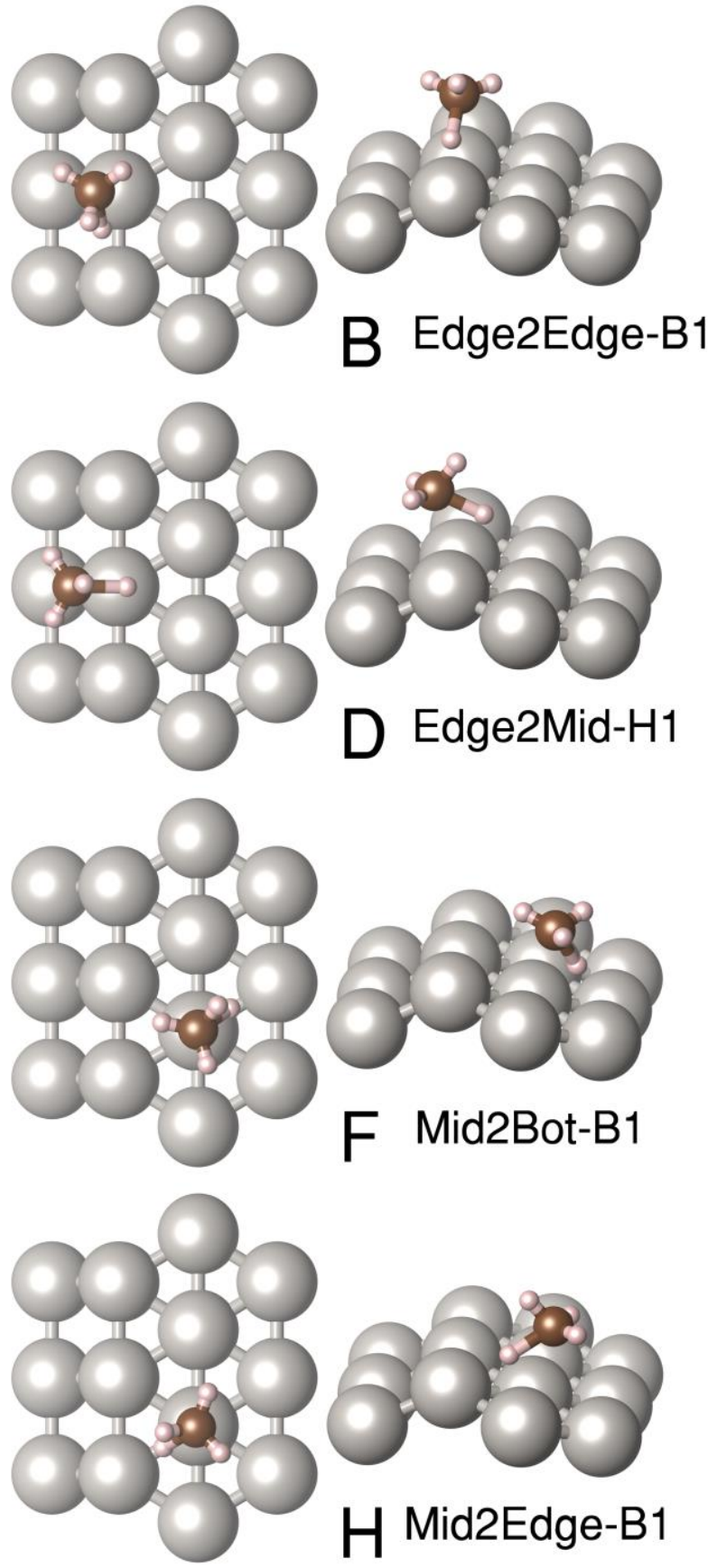

Figure 4. Depiction of the transition state geometries found in side and top view. TS A is the one found for $\mathrm{Pt}(111)$ and reported in Ref. 3. TSs B to H have been found on $\mathrm{Pt}(211)$ and are also labeled with the names reported in Table I. 

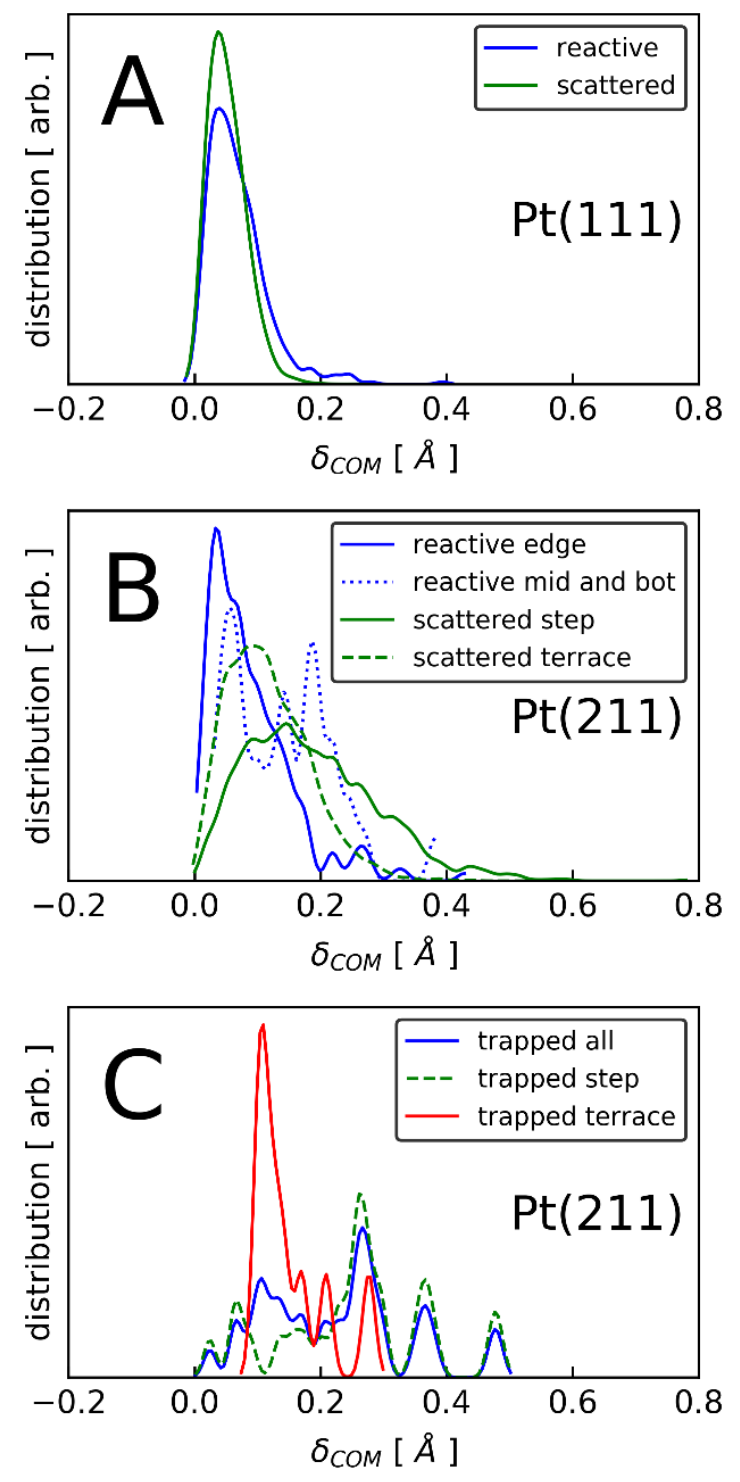

Figure 5. Panel A: $\delta_{\text {Сом }}$ computed for the reactive (blue) and scattered (green) trajectories on Pt(111). Panel B: $\delta_{\text {СOM }}$ computed for the reacted (blue) and scattered (green) trajectories on Pt(211), the solid lines describe step edge atom reaction and step facet scattering, the dotted line describes bottom and middle atom reactions and the dashed line describes terrace facet scattering. Panel C: $\delta_{C O M}$ computed on $\mathrm{Pt}(211)$ for all the trapped trajectories (blue) and for the trapped trajectories impacting first on the step (green) or on a terrace (red). 

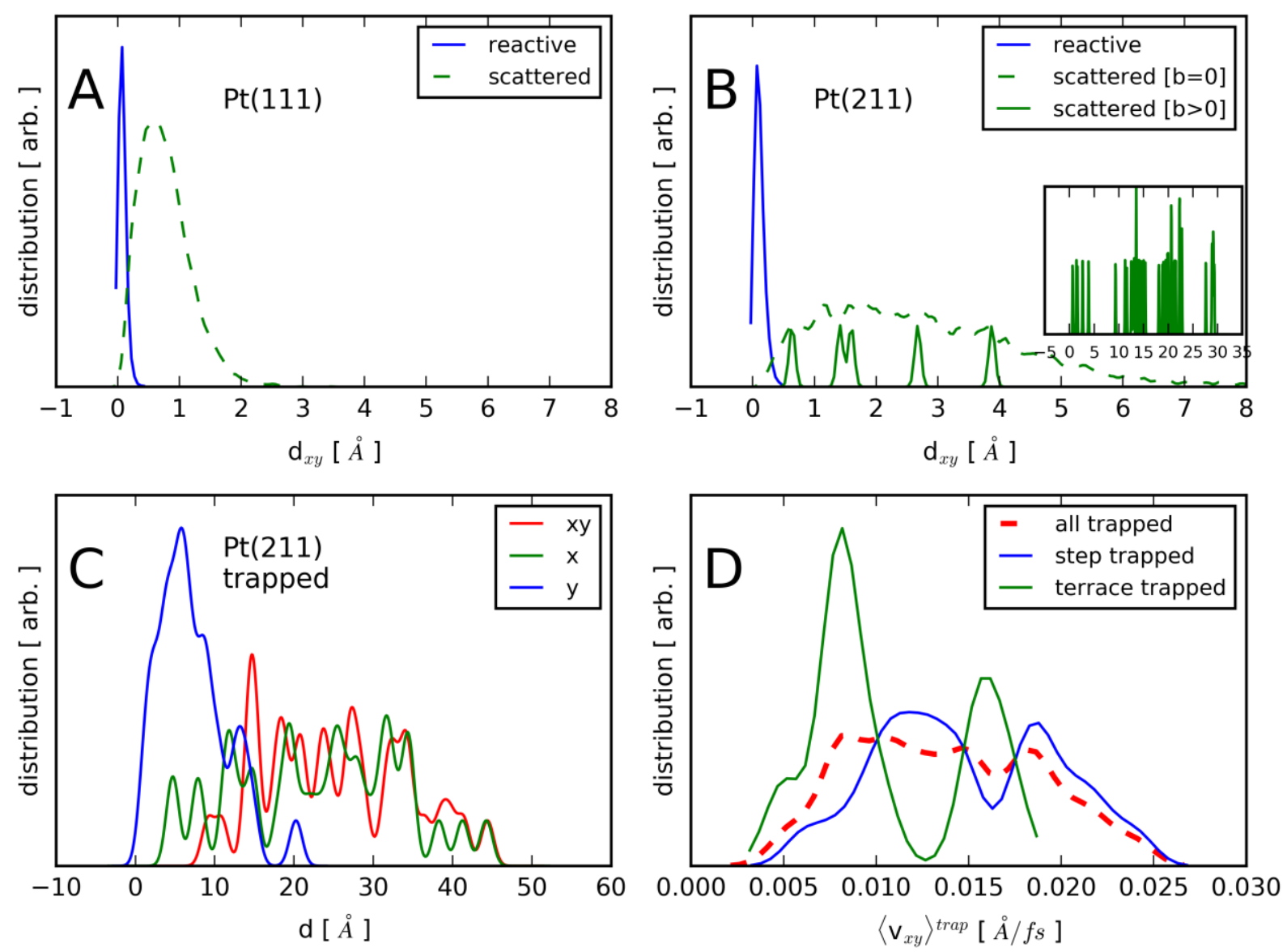

Figure 6. Panel A and B: distributions of the distance travelled by the molecules in the $x y$ plane $\left(d_{x y}\right)$ for reactive and scattered trajectories on the $\mathrm{Pt}(111)$ and $\mathrm{Pt}(211)$ surfaces, respectively. In Panels $\mathrm{A}$ and $\mathrm{B}$ the blue solid lines represent reactive trajectories (the distributions have been multiplied by 0.2 for a better comparison) and the green lines represent scattered trajectories without bouncing $(b=0$, dashed green) and with bouncing ( $b>0$, solid green) on the surface. Panel $c$ : distance travelled $(d)$ by the molecules trapped on the $\mathrm{Pt}(211)$ surface. The distance travelled in the $x$ direction, in the $y$ direction and the total distance travelled in the $x y$ plane are reported in green, blue and red respectively. Panel D: distribution of average COM velocity in the $x y$ plane for the trapped trajectories $\left\langle v_{x y}\right\rangle^{\text {trap }}$ for molecules that have the first impact on the terrace (green solid line), on the step (blue solid line) and for all the trapped molecules (red dashed line). 

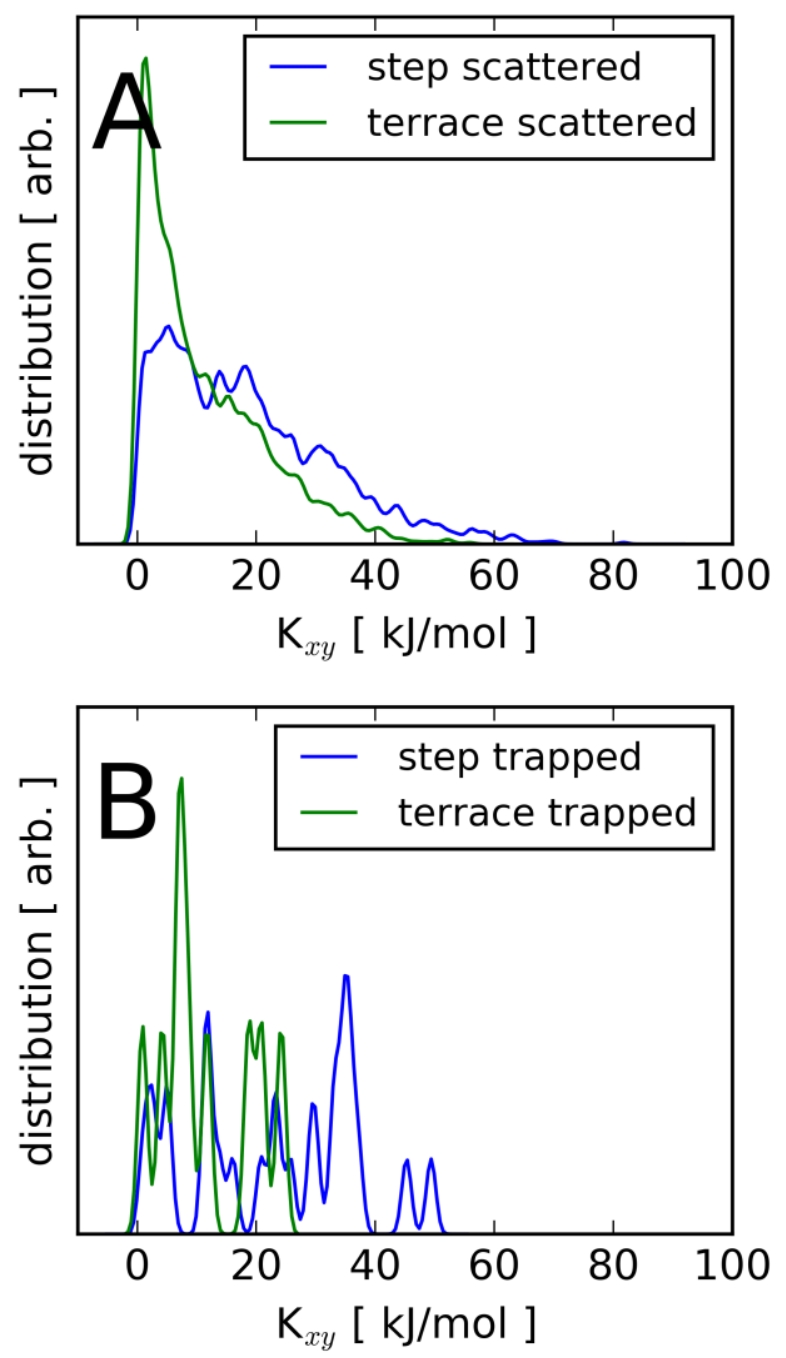

Figure 7. Distribution of kinetic energy in the $x y$ plane $\left(K_{x y}\right)$ after the impact with the $\mathrm{Pt}(211)$ surface for scattered and trapped molecules (Panel A and B, respectively) and for molecules that impacted on the step or on the terrace (blue and green, respectively). 


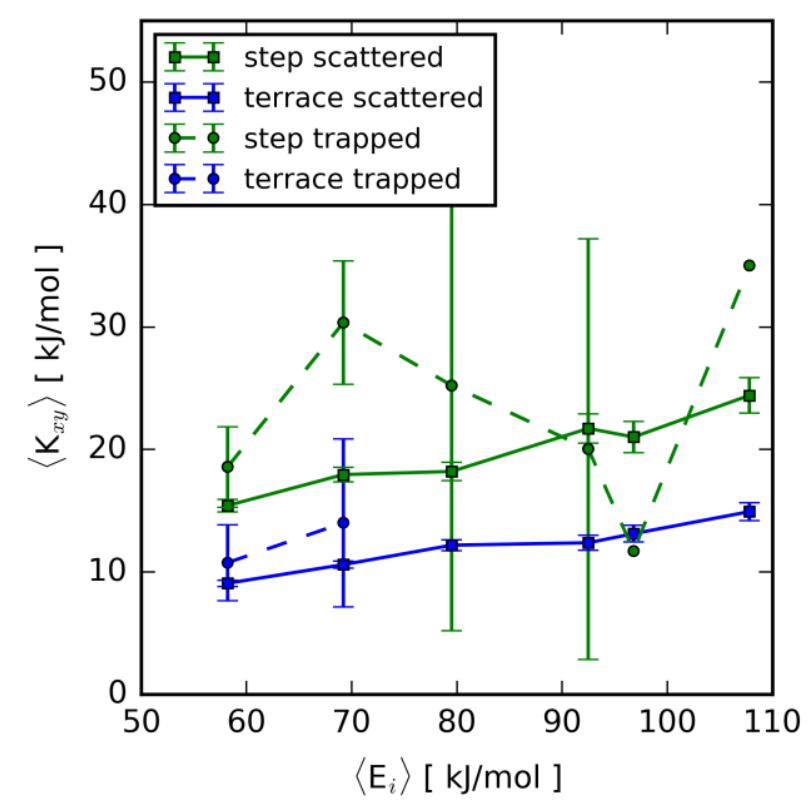

Figure 8. Average kinetic energy in the $x y$ plane $\left(\left\langle K_{x y}\right\rangle\right)$ as a function of the average initial kinetic energy $\left(\left\langle E_{i}\right\rangle\right)$ after the impact with the $\mathrm{Pt}(211)$ surface. Results for scattered molecules are reported as squares and solid lines and results for trapped molecules as circles and dashed lines. Green data represent molecules impacting on the step and blue data molecules impacting on the terrace. Two points are reported without error bars because the sample size for those data sets is 1 . The lines are drawn to guide the eye. 


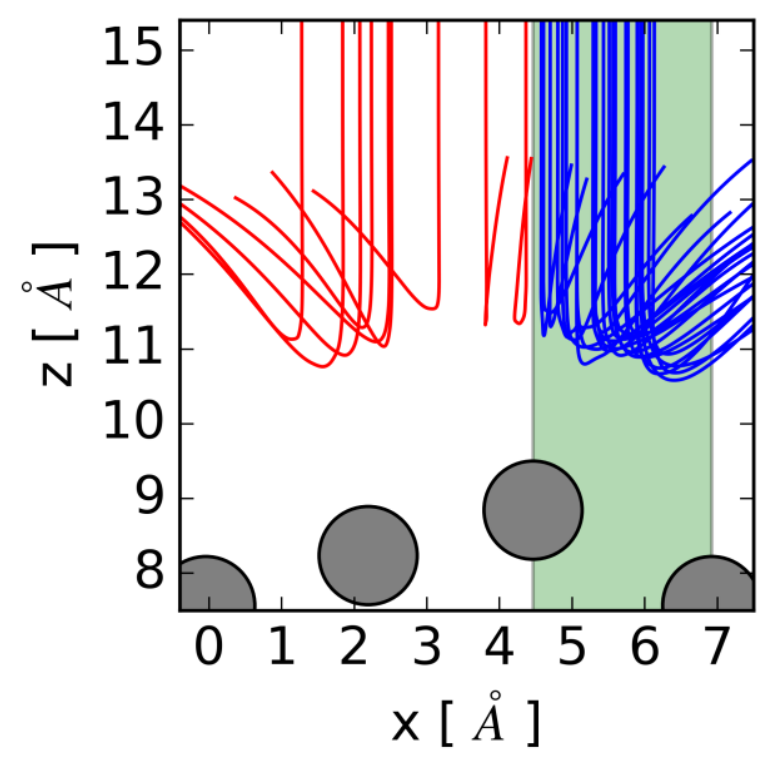

Figure 9. $x z$ projection of the first part of the trapped trajectories. The first layer atoms $0 \mathrm{~K}$ positions are reported as gray circles and the step is highlighted in green. The trajectories are reported in blue if the first impact is on the step and in red if it is on the terrace. 


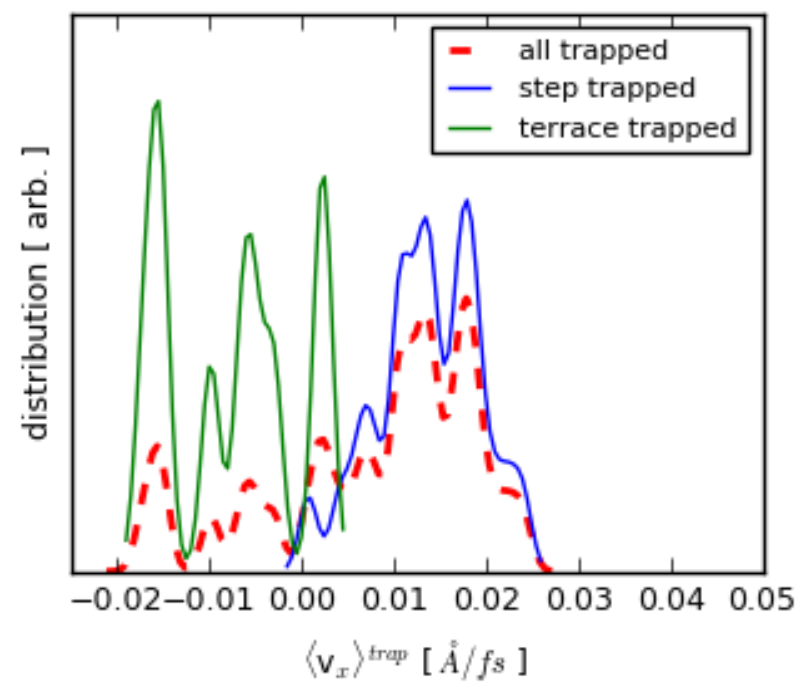

Figure 10. Distribution of the average velocity along $\mathrm{x}$ during trapping $\left\langle v_{x}\right\rangle^{\text {trap }}$ for all the trapped molecules (red line), the trapped molecules that have the first impact on the step (blue) and the ones that have the first impact on the terrace (green). 

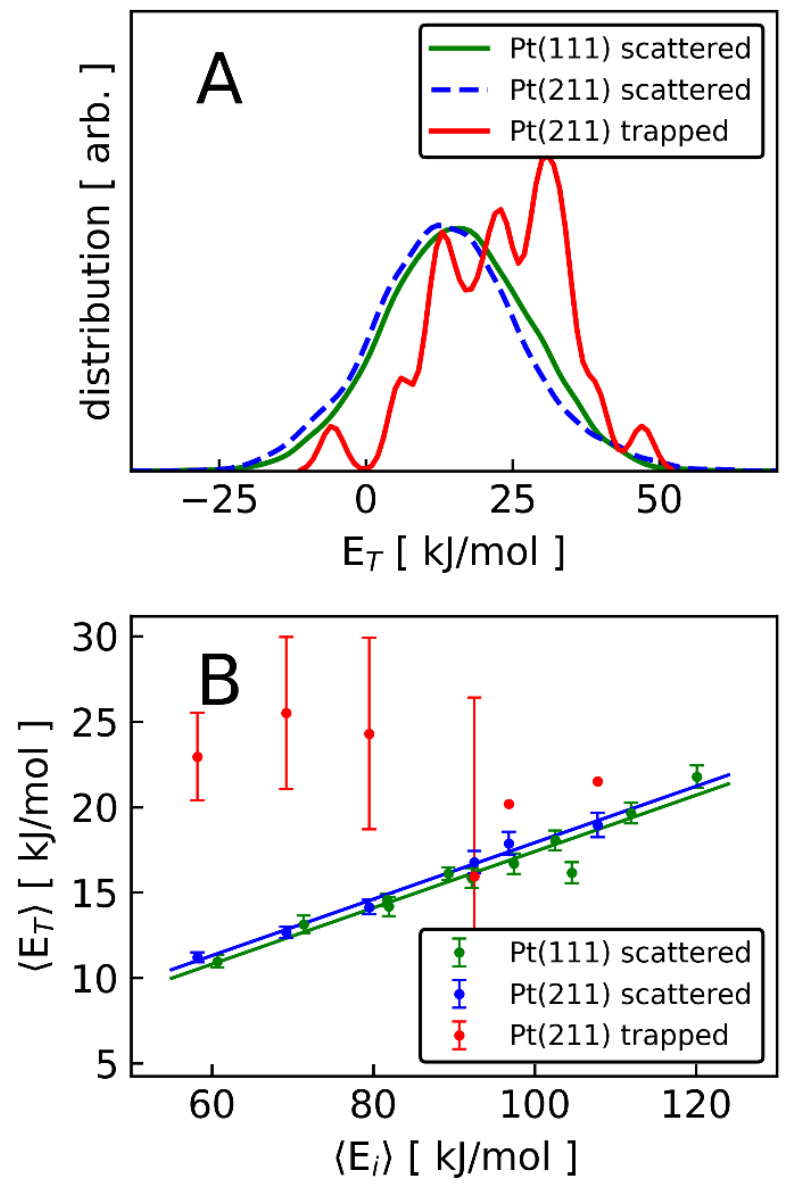

Figure 11. Panel A: energy transfer to the surface $\left(E_{T}\right)$. Results are reported for scattered molecules on the flat Pt(111) (green line), on the stepped Pt(211) (blue line) and for trapped molecules (after the first impact on the Pt(211) (red line). Panel B: average energy transfer to the surface versus average incident energy $\left(\left\langle E_{i}\right\rangle\right)$. Results are reported for scattered molecules on the flat $\mathrm{Pt}(111)$ (green), on the stepped Pt(211)) (blue) and for trapped molecules (after the first impact on the Pt(211)) (red). The lines are linear regressions of the data. Note that error bars are not shown for samples containing only one trapped trajectory. 

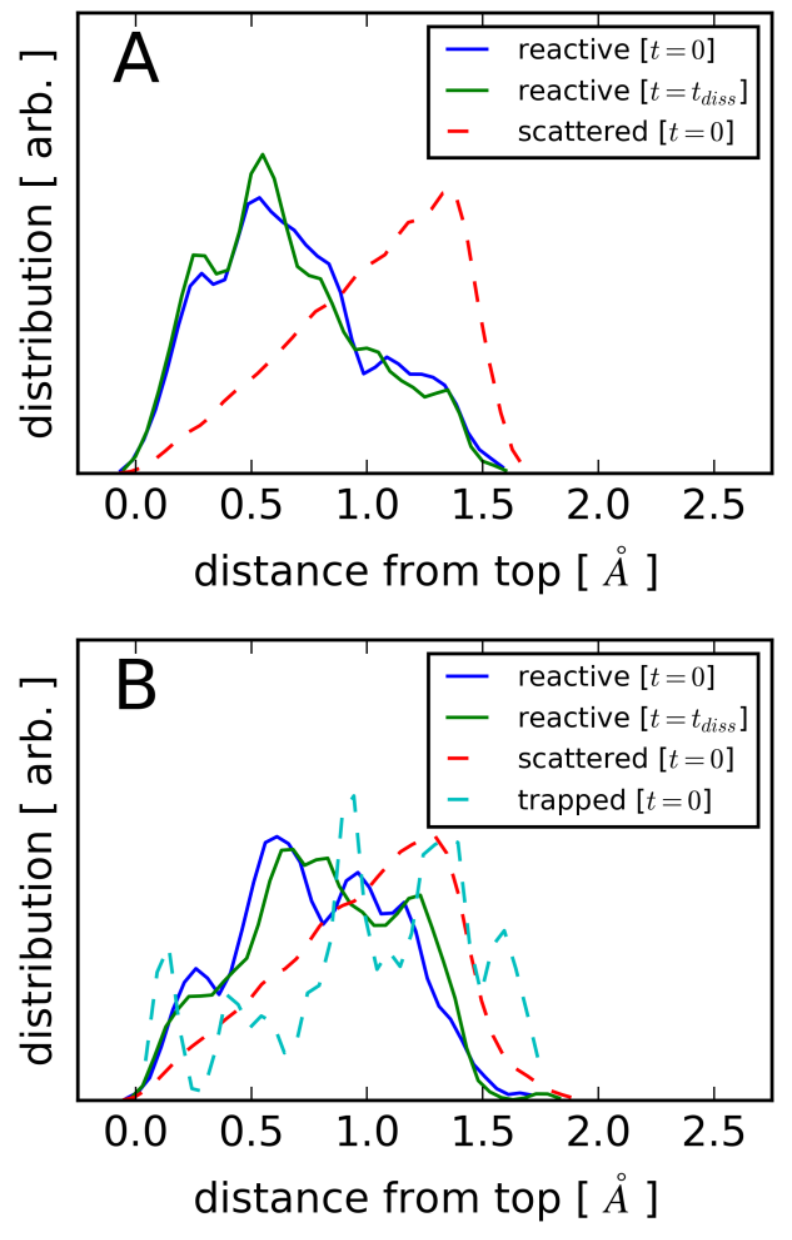

Figure 12. Distance from the closest top site in $x y$. In Panel A results for the Pt(111) surface and in Panel B for the Pt(211) surface are reported. Results for reactive trajectories are reported in blue and green for the initial position and the position at the dissociation time, respectively. Results for scattered and trapped trajectories are reported in red and cyan, respectively. 

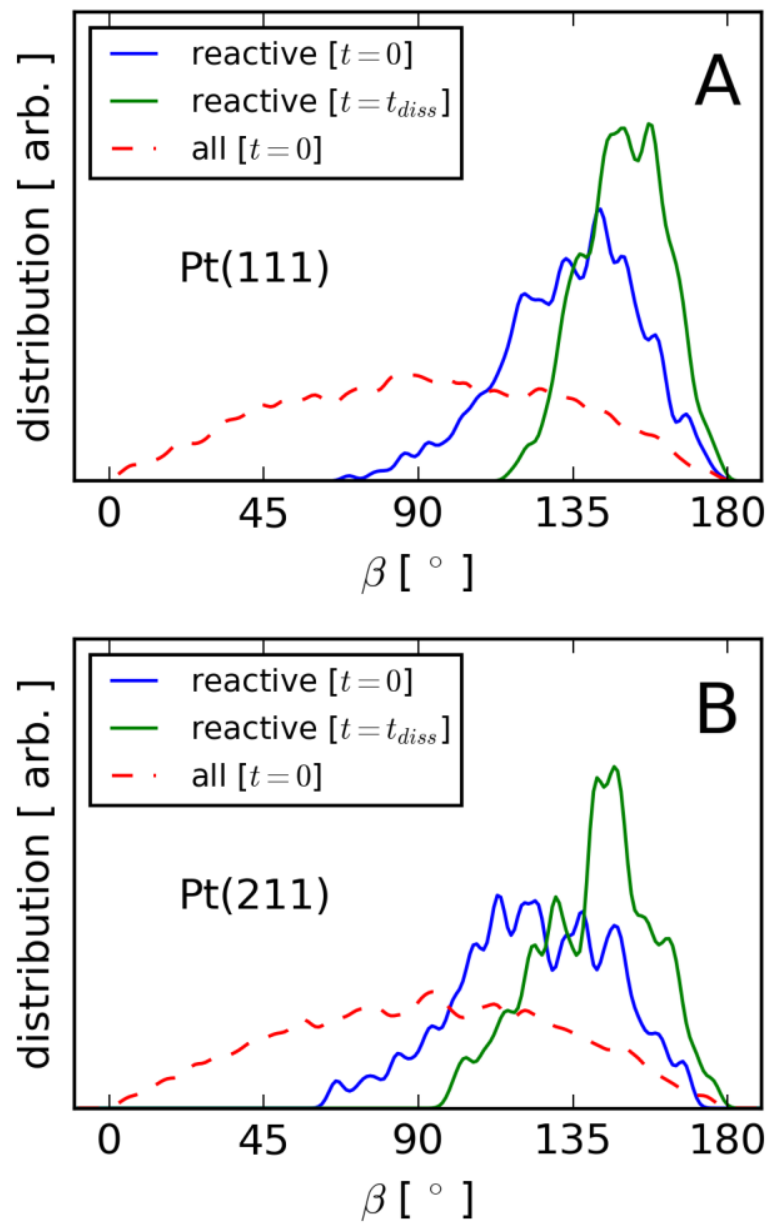

Figure 13. Panel A: $\beta$ distributions for $\mathrm{CHD}_{3}$ on $\mathrm{Pt}(111)$. Results for the reactive trajectories are reported in blue and green for the initial and the dissociation step, respectively. Results for the first time step of all the trajectories are reported in red. Panel B: same as Panel A but for Pt(211). 

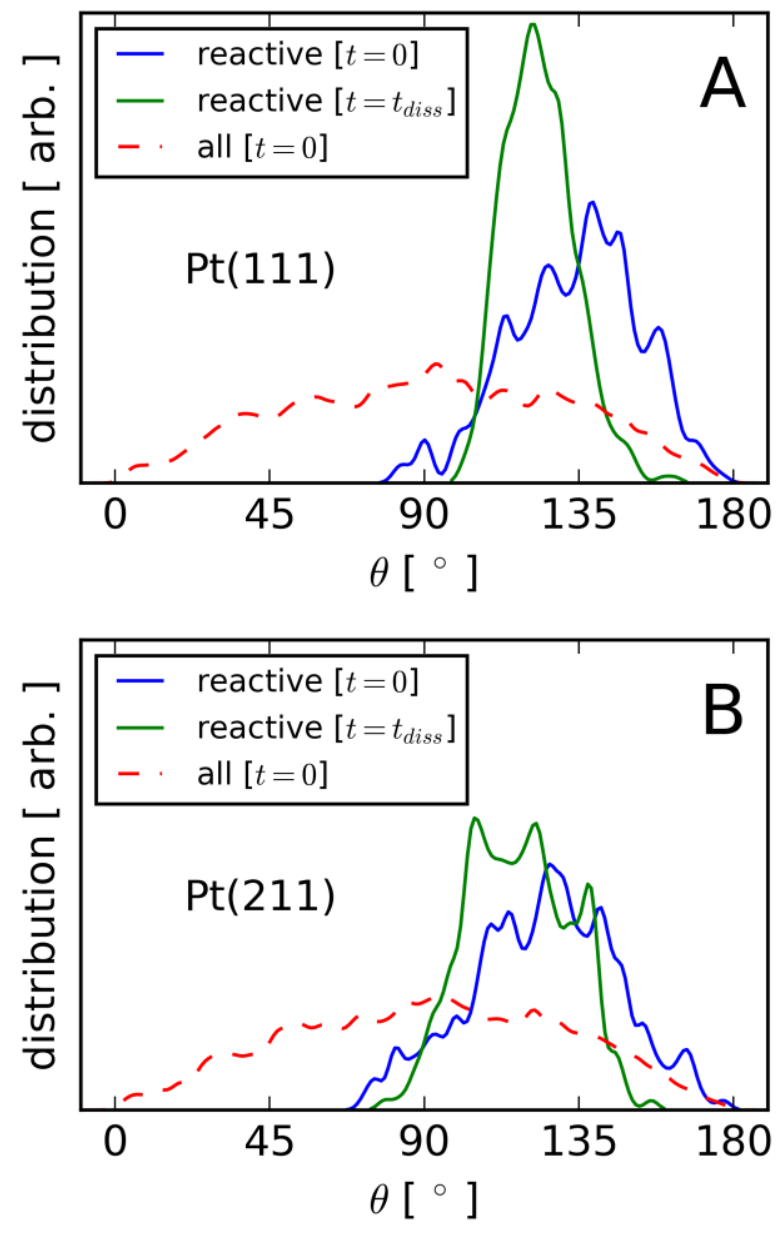

Figure 14. Same as Figure 13 but for $\theta$. 


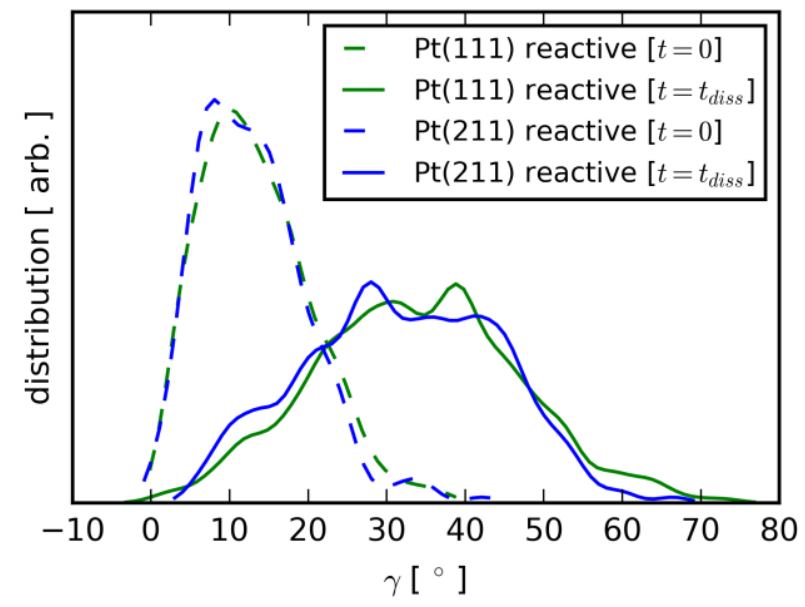

Figure 15. $\gamma$ distributions for $\mathrm{t}=0$ and for the dissociation step as dashed and solid lines, respectively. Green lines are for $\mathrm{Pt}(111)$ and blue lines $\mathrm{Pt}(211)$ results. 


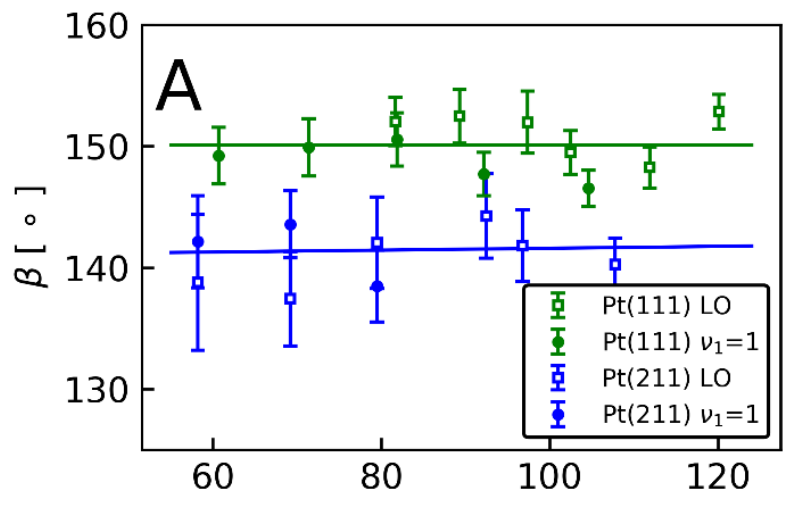

$\left\langle\mathrm{E}_{i}\right\rangle[\mathrm{kJ} / \mathrm{mol}]$
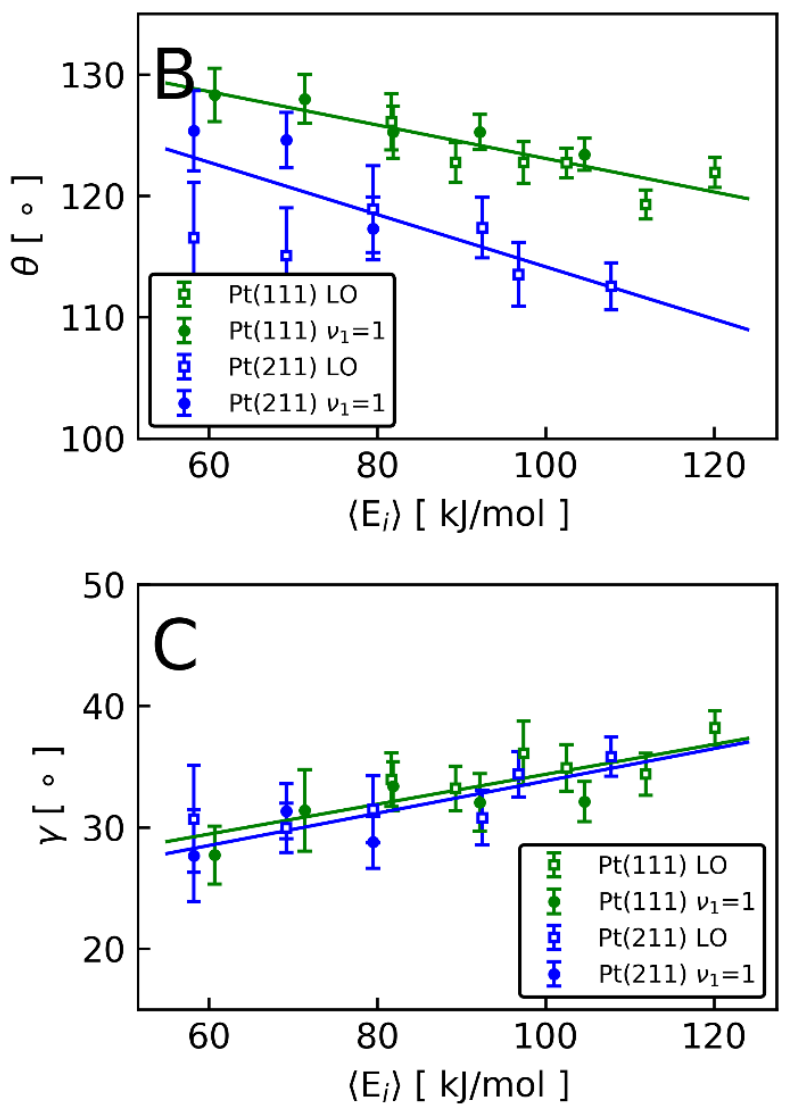

Figure 16. Dependence of $\beta, \theta$ and $\gamma$ on the initial average kinetic energy $\left\langle E_{i}\right\rangle$ at the moment of the dissociation. Results for the $\mathrm{Pt}(111)$ and for the $\mathrm{Pt}(211)$ are reported in green and blue, respectively. Open squares represent laser-off (LO) calculations and filled circles $v_{1}=1$ calculations. The linear regressions reported are computed considering both LO and $v_{1}=1$ trajectories. 


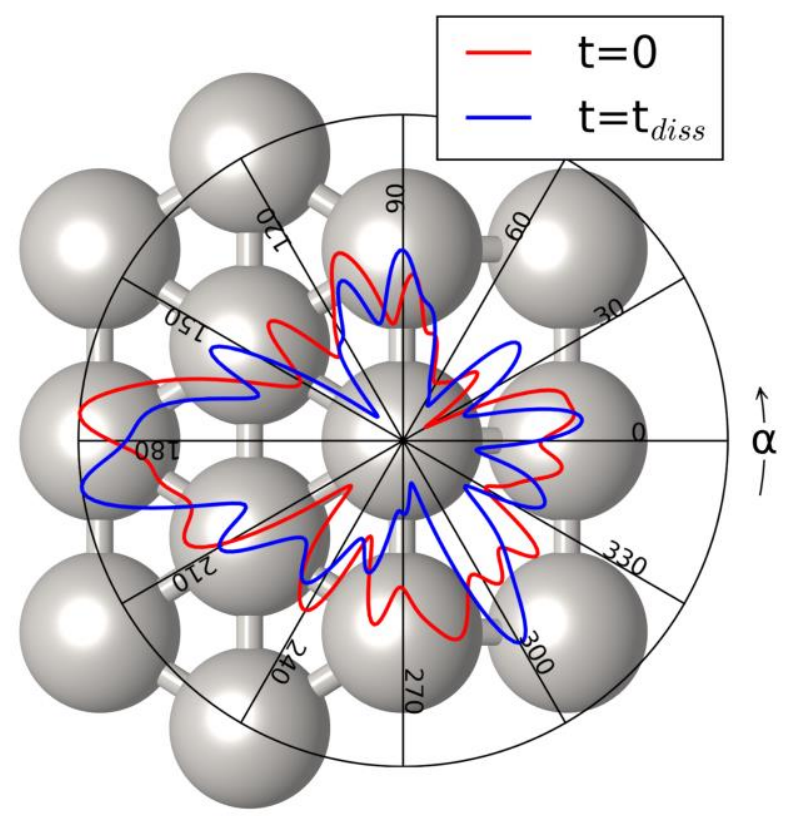

Figure 17. Distribution of $\alpha$ at $\mathrm{t}=0$ and at the time of the dissociation ( $\mathrm{t}_{\text {diss }}$ ) (in red and blue, respectively) for molecules that reacted on the step edge atom. The value of $\alpha$ is reported in black and it increases counter-clockwise. The plot is superimposed on the $\mathrm{Pt}(211) 1^{\text {st }}$ layer top view. The closer the distribution is to the external black circle the more that value of $\alpha$ is represented. 


\section{References}

${ }^{1}$ C. A. Wolcott, A. J. Medford, F. Studt, and C. T. Campbell, J. Catal. 330, 197 (2015).

${ }^{2}$ Y. Xu, A. C. Lausche, S. Wang, T. S. Khan, F. Abild-Pedersen, F. Studt, J. K. Nørskov, and T. Bligaard, New J. Phys. 15, 125021 (2013).

${ }^{3}$ D. Migliorini, H. Chadwick, F. Nattino, A. Gutiérrez-González, E. Dombrowski, E. A. High, H. Guo, A. L. Utz, B. Jackson, R. D. Beck, and G. J. Kroes, J. Phys. Chem. Lett. 8, 4177 (2017).

${ }^{4}$ G. Füchsel, P. S. Thomas, J. den Uyl, Y. Öztürk, F. Nattino, H. D. Meyer, and G. J. Kroes, Phys. Chem. Chem. Phys. 18, 8174 (2016).

${ }^{5}$ F. Nattino, D. Migliorini, G. J. Kroes, E. Dombrowski, E. A. High, D. R. Killelea, and A. L. Utz, J. Phys. Chem. Lett. 7, 2402 (2016).

${ }^{6}$ F. Nattino, D. Migliorini, M. Bonfanti, and G. J. Kroes, J. Chem. Phys. 144, 044702 (2016).

${ }^{7}$ H. Guo and B. Jackson, J. Phys. Chem. C 119, 14769 (2015).

${ }^{8}$ S. Nave and B. Jackson, Phys. Rev. Lett. 98, 173003 (2007).

${ }^{9}$ A. K. Tiwari, S. Nave, and B. Jackson, J. Chem. Phys. 132, 134702 (2010).

${ }^{10}$ X. Shen, Z. Zhang, and D. H. Zhang, J. Chem. Phys. 144, 101101 (2016).

${ }^{11}$ R. R. Smith, D. R. Killelea, D. F. DelSesto, and A. L. Utz, Science 304, 992 (2004).

${ }^{12}$ D. R. Killelea, V. L. Campbell, N. S. Shuman, and A. L. Utz, Science 319, 790 (2008).

${ }^{13}$ R. D. Beck, P. Maroni, D. C. Papageorgopoulos, T. T. Dang, M. P. Schmid, T. R. Rizzo, Science 302, 98 (2003).

${ }^{14}$ B. L. Yoder, R. Bisson, and R. D. Beck, Science 329, 553 (2010).

15 J. Wei and E. Iglesia, J. Phys. Chem. B 108, 4094 (2004).

${ }^{16}$ B. Jiang, R. Liu, J. Li, D. Xie, M. Yang, and H. Guo, Chem. Sci. 4, 3249 (2013).

${ }^{17}$ X. Shen, J. Chen, Z. Zhang, K. Shao, and D. H. Zhang, J. Chem. Phys. 143, 144701 (2015).

${ }^{18}$ P. M. Hundt, B. Jiang, M. E. van Reijzen, H. Guo, and R. D. Beck, Science 344, 504 (2014).

${ }^{19}$ S. Nave and B. Jackson, Phys. Rev. B - Condens. Matter Mater. Phys. 81, 233408 (2010).

${ }^{20}$ B. Jackson and S. Nave, J. Chem. Phys. 138, 174705 (2013).

${ }^{21}$ A. Farjamnia and B. Jackson, J. Chem. Phys. 142, 234705 (2015).

${ }^{22}$ G. J. Kroes, J. Phys. Chem. Lett. 6, 4106 (2015).

${ }^{23}$ C. Díaz, E. Pijper, R. A. Olsen, H. F. Busnengo, D. J. Auerbach, and G. J. Kroes, Science 326, 832 (2009).

${ }^{24}$ L. Sementa, M. Wijzenbroek, B. J. van Kolck, M. F. Somers, A. Al-Halabi, H. F. Busnengo, R. A. Olsen, G. J. Kroes, M. Rutkowski, C. Thewes, N. F. Kleimeier, and H. Zacharias, J. Chem. Phys. 138, 044708 (2013). 
${ }^{25}$ E. Nour Ghassemi, M. Wijzenbroek, M. F. Somers, and G. J. Kroes, Chem. Phys. Lett. 683, 329 (2017).

${ }^{26}$ Y. Y. Chuang, M. L. Radhakrishnan, P. L. Fast, C. J. Cramer, and D. G. Truhlar, J. Phys. Chem. A 103, 4893 (1999).

${ }^{27}$ B. Hammer, L. B. Hansen, and J. K. Nørskov, Phys. Rev. B 59, 7413 (1999).

28 J. P. Perdew, K. Burke, and M. Ernzerhof, Phys. Rev. Lett. 77, 3865 (1996).

${ }^{29}$ J. P. Perdew, K. Burke, and M. Ernzerhof, Phys. Rev. Lett. 78, 1396 (1997).

${ }^{30}$ G. Román-Pérez and J. M. Soler, Phys. Rev. Lett. 103, 096102 (2009).

${ }^{31}$ M. Dion, H. Rydberg, E. Schröder, D. C. Langreth, and B. I. Lundqvist, Phys. Rev. Lett. 92, 246401 (2004).

${ }^{32}$ H. S. Taylor, Proc. R. Soc. London. Ser. A 108, 105 (1925).

${ }^{33}$ T. Zambelli, J. Wintterlin, J. Trost, and G. Ertl, Science 273, 1688 (1996).

${ }^{34}$ B. Hammer, Phys. Rev. Lett. 83, 3681 (1999).

${ }^{35}$ A. T. Gee, B.E. Hayden, C. Mormiche, A. W. Kleyn, and B. Riedmüller, J. Chem. Phys. 118, 3334 (2003).

${ }^{36}$ F. Abild-Pedersen, O. Lytken, J. Engbæk, G. Nielsen, I. Chorkendorff, and J. K. Nørskov, Surf. Sci. 590, 127 (2005).

${ }^{37}$ G. Kresse and J. Hafner, Phys. Rev. B 49, 14251 (1994).

${ }^{38}$ G. Kresse and J. Furthmüller, Phys. Rev. B 54, 11169 (1996).

${ }^{39}$ G. Kresse and J. Furthmüller, Comput. Mater. Sci. 6, 15 (1996).

${ }^{40}$ G. Kresse and J. Hafner, Phys. Rev. B 47, 558 (1993).

${ }^{41}$ P. E. Blöchl, Phys. Rev. B 50, 17953 (1994).

${ }^{42}$ G. Kresse and D. Joubert, Phys. Rev. B 59, 1758 (1999).

${ }^{43}$ H. Chadwick, A. Gutiérrez-González, and R. D. Beck, J. Chem. Phys. 145, 174707 (2016).

${ }^{44}$ P. Xiao, D. Sheppard, J. Rogal, and G. Henkelman, J. Chem. Phys. 140, 174104 (2014).

45 J. Kästner and P. Sherwood, J. Chem. Phys. 128, 014106 (2008).

${ }^{46}$ A. Heyden, A. T. Bell, and F. J. Keil, J. Chem. Phys. 123, 224101 (2005).

${ }^{47}$ G. Henkelman and H. Jónsson, J. Chem. Phys. 111, 7010 (1999).

${ }^{48}$ S. Nave, A.K. Tiwari, and B. Jackson, J. Chem. Phys. 132, 054705 (2010). 